\title{
Esophageal injury during radiofrequency ablation for atrial fibrillation
}

\author{
A. Marc Gillinov, MD, Gosta Pettersson, MD, and Thomas W. Rice, MD, Cleveland, Ohio
}

$\mathrm{R}$ ecent reports document successful operative treatment of atrial fibrillation by radiofrequency ablation of the left atrium. ${ }^{1}$ We report the case of a patient with rheumatic mitral valve disease and chronic atrial fibrillation who had a fatal esophageal injury during radiofrequency ablation of the left atrium.

\section{Clinical Summary}

A 60-year-old woman with rheumatic mitral valve disease and chronic atrial fibrillation of several years' duration came for surgical evaluation. The patient was small and cachectic, with a weight of 29 $\mathrm{kg}$, a height of $146 \mathrm{~cm}$, and a body surface area of $1.07 \mathrm{~m}^{2}$. Transesophageal echocardiography demonstrated normal left and right ventricular function, biatrial enlargement (maximum left atrial diameter $7.0 \mathrm{~cm}$ ), 2+ mitral regurgitation, and severe mitral stenosis.

She was taken to the operating room on April 30, 2001. A transesophageal echocardiogram was performed, and then the echo probe was removed. A partial upper sternotomy was constructed and the mitral valve exposed with a combined superior and transseptal approach to the left atrium. The mitral valve was replaced with a 25 mm Carpentier-Edwards Perimount valve (Edwards Lifesciences, LLC, Newport Beach, Calif). The Cobra RF System (Boston Scientific, Boston, Mass) was then used to create a series of radiofrequency lesions in the left atrium. An oval lesion was created around the left pulmonary veins, and a similar lesion was placed around the right pulmonary veins. These two lesions were connected by a transverse lesion across the back wall of the left atrium. A lesion was created around the left atrial appendage. Additional lesions were created from the left atrial appendage and from the posterior mitral anulus to the left pulmonary vein encircling lesion. All lesions were created at a temperature of $80^{\circ} \mathrm{C}$ for 60 seconds. The left atrial appendage was excised.

The patient left the operating room with atrioventricular pacing and underlying nodal rhythm. Over the ensuing 10 days she was in sinus rhythm most of the time but had intermittent atrial fibrillation

\footnotetext{
From the Department of Thoracic and Cardiovascular Surgery, The Cleveland Clinic Foundation, Cleveland, Ohio.

Received for publication May 31, 2001; accepted for publication June 12, 2001.

Address for reprints: A. Marc Gillinov, MD, Department of Thoracic and Cardiovascular Surgery/F24, The Cleveland Clinic Foundation, 9500 Euclid Ave, Cleveland, OH 44195 (E-mail: gillinom@ccf.org).

J Thorac Cardiovasc Surg 2001;122:1239-40

Copyright $\odot 2001$ by The American Association for Thoracic Surgery

0022-5223/2001 \$35.00+ $0 \quad \mathbf{1 2 / 5 4 / 1 1 8 0 4 1}$

doi:10.1067/mtc.2001.118041
}

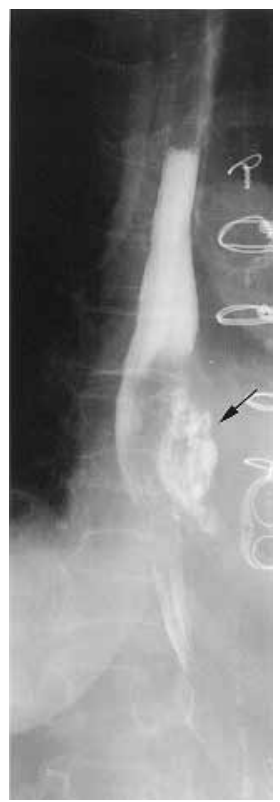

Figure 1. Esophagogram demonstrating extravasation of contrast medium (arrow) at the level of the carina.

that was treated with amiodarone and electrical cardioversion. Immediately postoperatively she had an elevated white blood cell count $\left(27,000\right.$ cells $\left./ \mathrm{mm}^{3}\right)$. Although the white blood cell count decreased, it never normalized. On postoperative day 9 the white count rose to 36,000 cells $/ \mathrm{mm}^{3}$ and odynophagia developed. A computed tomographic scan of the chest demonstrated mediastinal air at the level of the carina. Water-soluble contrast medium and thin barium esophagograms performed on postoperative day 10 revealed a contained esophageal leak at the level of the left atrium (Figure 1).

The patient was returned to the operating room within hours of the diagnosis. Esophagoscopy confirmed esophageal perforation at $27 \mathrm{~cm}$ from the incisors. A right thoracotomy disclosed marked periesophageal inflammation that contained the leak in the mediastinum. Two linear perforations were found on the anterior wall of the esophagus behind the carina. During the esophageal dissection, cardiac tamponade and circulatory collapse ensued. The tamponade was relieved, but the patient required emergency cardiopulmonary bypass for hemodynamic support. The manipulation of the heart had provoked bleeding from a temporary pacing wire site in the right atrium. There was no bleeding from the left atrium. The esophagectomy was completed and the patient weaned from bypass. Over the ensuing hours, overwhelming sepsis, severe pulmonary dysfunction, and uncorrectable coagulopathy developed. The patient died the following morning. Pathologic examination of 
the esophagus demonstrated anterior esophageal perforation with transmural necrosis and granulation tissue.

\section{Discussion}

The combination of the Cox maze procedure and mitral valve surgery in patients with chronic atrial fibrillation and mitral valve disease cures atrial fibrillation in more than $70 \%$ of patients. ${ }^{2,3}$ Although efficacy and safety are well established, the technical complexity of the Cox maze procedure has prevented widespread adoption by surgeons.

In $75 \%$ of patients with atrial fibrillation and mitral valve disease, the atrial fibrillation is anchored in the left atrium. ${ }^{4}$ Therefore, a controlled pattern of left atrial lesions is likely to cure atrial fibrillation in a substantial proportion of such patients. With radiofrequency ablation of atrial fibrillation, the goal is to create a series of transmural lesions in the left atrium. The probe is set to deliver energy to achieve a preselected temperature for a predetermined duration. Although the temperature and duration of energy delivery are easily controlled, the lesion depth is not; thus, it is possible to create nontransmural lesions resulting in an unsuccessful operation. More important, this limitation introduces the possibility of creating a lesion that is too deep with consequent injury to adjacent structures. Mohr and colleagues, ${ }^{5}$ at the 2001 Annual Meeting of The American Association for Thoracic Surgery, reported a fatal esophageal injury with a left atrial-esophageal fistula after radiofrequency ablation of atrial fibrillation. It has been suggested that this injury was related to the transesophageal echocardiographic probe and that removal of the probe before delivery of radiofrequency energy should prevent esophageal injury. Clearly, this is not the case in our patient; the transesophageal probe was removed before radiofrequency ablation.
In this report, we describe a fatal complication associated with delivery of radiofrequency energy to treat atrial fibrillation. The patient was extremely small and cachectic, and it is likely that she had a thin left atrium with very little tissue between the left atrium and the esophagus; these factors may have potentiated the esophageal injury. Many surgeons would embrace a greatly simplified procedure to treat atrial fibrillation, even if its efficacy was slightly reduced. However, left atrial radiofrequency ablation for treatment of atrial fibrillation should be used with caution in very thin persons, and surgeons must be cognizant of the potential for esophageal injury with this procedure.

\section{References}

1. Alfieri O, Benussi S. Mitral valve surgery with concomitant treatment of atrial fibrillation. Cardiol Rev. 2000;8:317-21.

2. Handa N, Schaff HV, Morris JJ, Anderson BJ, Kopecky SL, EnriquesSarano M. Outcome of valve repair and the Cox maze procedure for mitral regurgitation and associated atrial fibrillation. J Thorac Cardiovasc Surg. 1999;118:628-35.

3. Cox JL, Ad N, Palazzo T. Impact of the maze procedure on the stroke rate in patients with atrial fibrillation. J Thorac Cardiovasc Surg. 1999;118:833-40.

4. Kottkamp H, Hindricks G, Hammel D, Autschbach R, Mergenthaler J, Borggrefe M, et al. Intraoperative radiofrequency ablation of chronic atrial fibrillation: a left atrial curative approach by elimination of anatomic "anchor" reentrant circuits. J Cardiovasc Electrophysiol. 1999;10:772-80.

5. Mohr FW, Nikolaus D, Falk V, Walther T, Hindricks G, Kottkamp H, et al. Curative treatment of atrial fibrillation: acute and midterm results of intraoperative radiofrequency ablation of atrial fibrillation in 150 patients. Read at the Eighty-first Annual Meeting of The American Association for Thoracic Surgery, May 7, 2001. 